\title{
Fouilles récentes dans la zone sacrée d'Istros
}

\section{Alexandru Avram et lulian Bîrzescu}

\section{OpenEdition}

Journals

Édition électronique

URL : http://journals.openedition.org/edl/362

DOI : $10.4000 /$ edl.362

ISSN : 2296-5084

\section{Éditeur}

Université de Lausanne

\section{Édition imprimée}

Date de publication : 15 mai 2012

Pagination : 279-310

ISBN : 978-2-940331-27-7

ISSN : 0014-2026

\section{Référence électronique}

Alexandru Avram et lulian Bîrzescu, « Fouilles récentes dans la zone sacrée d'Istros », Études de lettres [En ligne], 1-2 | 2012, mis en ligne le 15 mai 2015, consulté le 21 décembre 2020. URL : http:// journals.openedition.org/edl/362; DOI : https://doi.org/10.4000/edl.362 


\section{FOUILLES RÉCENTES DANS LA ZONE SACRÉE D'ISTROS}

Les auteurs présentent les acquis des nouvelles recherches dans le téménos d'Istros (Histria, Roumanie). Après un bref aperçu des résultats des anciennes fouilles (voir Histria VII), ils discutent notamment la "fosse sacrée» identifiée à l'est du temple d'Aphrodite et un nouvel édifice cultuel du dernier tiers du VI e s. av. J.-C. (monument $M$ ), détruit pendant la première moitié du $\mathrm{V}^{\mathrm{e}}$ siècle av. J.-C. et qui pourrait être interprété comme le temple d'une divinité féminine. Ils ajoutent des considérations sur les premiers aménagements cultuels ayant précédé la construction des temples en pierre, ainsi que sur les changements qu'a connus successivement le téménos aux époques classique et hellénistique. Seules les trouvailles jugées les plus significatives sont illustrées.

\section{Introduction}

L'histoire d'Istros (ou Histria, de son nom latin) s'échelonne sur plus de 1300 ans, depuis sa fondation par Milet vers le milieu du VII s. av. J.-C. jusqu'à l'abandon de la ville après la perte du contrôle sur le Danube par l'Empire byzantin en 602 apr. J.-C. (fig. 1). Des événements, parfois dramatiques, appartenant tantôt à l'histoire universelle, tantôt à l'histoire locale, ont parsemé ce parcours. Hasard ou non, les grandes divisions de l'histoire universelle coïncident à Istros avec les étapes de son histoire locale: car la fin de chaque cycle est marquée par une grande destruction, suivie par une reconstruction, dont la portée est différente selon le cas. La reconstitution de l'histoire de la cité doit, certes, beaucoup à la superbe moisson de plus de 400 inscriptions (ISM I) ${ }^{1}$. Elle tient

I. Voir, plus récemment, A. Avram, «Le corpus des inscriptions d'Istros revisité». 
280

ÉTUDES DE LETTRES

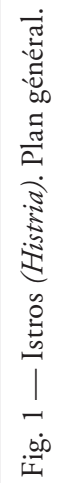


également compte des quelques sources littéraires que les Anciens nous ont léguées, mais elle repose essentiellement sur le traitement des données archéologiques patiemment accumulées depuis 1914, lorsque Vasile Pârvan, le fondateur de l'archéologie classique en Roumanie, avait ouvert la première campagne de fouilles à Istros. A la seule exception des années des deux guerres mondiales et de la période d'après-guerre, des campagnes de fouilles y eurent lieu depuis lors chaque année et se poursuivent encore à l'heure qu'il est sous la direction de l'Institut d'Archéologie de Bucarest $^{2}$.

Le téménos d'Istros, communément désigné dans le langage des archéologues locaux comme "Zone Sacrée», a toujours constitué le point central d'intérêt des fouilleurs ${ }^{3}$. Comme il est d'ailleurs normal, toute l'histoire de la cité se concentre dans l'évolution de son téménos, et c'est à partir des données fournies par ces fouilles que les grandes étapes, dont il sera question plus bas, ont pu être définies.

\section{Description générale de la Zone Sacrée}

La Zone Sacrée d'Istros est située dans le secteur NE de la ville, sur "l'acropole" occupant un rocher de schiste au bord du lac de Sinoé, ancien golfe de la mer Noire. Il s'agit plus exactement de la ville haute, opposée à l'habitat civil, lequel s'étendait plus à l'ouest, sur le "plateau ".

Le paysage antique différait beaucoup de ce qui se présente aujourd'hui aux yeux de ceux qui prennent le plaisir de visiter les ruines de l'ancienne cité milésienne (pl. XXXV). Des recherches géomorphologiques et archéomagnétiques, poursuivies à plusieurs reprises, ont démontré qu'une bonne partie de cette "acropole" a été engloutie par les eaux de la mer à la suite d'une transgression dont on date les débuts vers la fin de l'ère païenne. Vers la fin du III' s. apr. J.-C., lors de la construction d'un nouveau rempart après la destruction de la ville par les Goths (vers 251 ?), son segment est, dont nous venons de découvrir un tronçon

2. Voir, pour quelques aperçus généraux, P. Alexandrescu, W. Schuller (Hrsg.), Histra (avec, à la fin, une bibliographie raisonnée compilée par A. Avram) et A. Avram, «Histria».

3. Pour les différentes étapes de l'histoire des fouilles, voir K. Zimmermann, "Ausgrabungen in der Tempelzone von Histria» et "Griechische Altäre in der Tempelzone von Histria». 
pendant les dernières campagnes, passait déjà sur des restes de monuments de l'ancienne Zone Sacrée. La limite orientale initiale du téménos demeure donc inconnue ${ }^{4}$.

Les recherches menées jusqu'en 1989 ont été publiées en 2005 dans un volume monumental par notre maître Petre Alexandrescu ${ }^{5}$, dont nous déplorons la disparition. Nous y renvoyons pour des détails, sans oublier, bien entendu, deux ouvrages quasiment complémentaires consacrés respectivement à la sculpture en pierre ${ }^{6}$ et aux membra disiecta en provenance d'ensembles architecturaux d'Istros ${ }^{7}$. S'il n'est, certes, pas question de reprendre ici toute la richesse de données accumulées pendant des décennies de recherches, il convient pour autant d'en donner un bref résumé.

Les origines du téménos remontent sans aucun doute à la fondation même de la cité. Les premiers indices d'aménagements cultuels sont fournis par quelques petites fosses datées de la fin du VII et du début du $\mathrm{VI}^{\mathrm{e}}$ s. av. J.-C. Parmi ces fosses, il y en a une, creusée dans le rocher, qui était sûrement un bothros, vu sa position à proximité de l'angle NO d'un temple que l'on est à même d'attribuer à Apollon Iètros, principale divinité de la cité ${ }^{8}$. D'autre part, les dates assignées à quelques terres cuites architecturales, et particulièrement à la tuile portant une dédicace à Aphrodite (premier quart du $\mathrm{VI}^{\mathrm{e}}$ s. av. J.-C.) ${ }^{9}$, suggèrent l'existence d'une première série de bâtiments cultuels construits en bois. L'un d'eux aurait été l'oikos d'Aphrodite, ayant précédé le futur temple en pierre.

Les premiers édifices en pierre, et plus exactement en grès calcaire, datent du début du troisième quart $\mathrm{du} \mathrm{VI}^{\mathrm{e}}$ s. av. J.-C. et leurs phases

4. Pour la topographie d'Istros, voir notamment P. Alexandrescu, «Notes de topographie histrienne" = L'Aigle et le dauphin, p. 47-63; O. Höckmann, G. J. Peschel, A. Woehl, "Zur Lage des Hafens von Histria». Pour l'urbanisme d'Istros, l'étude de M. Mărgineanu Cârstoiu, «Plans de villes romaines en Mésie inférieure», demeure fondamentale.

5. P. Alexandrescu et al., Histria VII.

6. M. Alexandrescu Vianu, Histria IX.

7. M. Mărgineanu Cârstoiu, Histria XII. Nous mentionnons en outre deux autres contributions récentes: A. Avram, K. Zimmermann, M. Mărgineanu Cârstoiu, I. Bîrzescu, «Nouvelles données sur la Zone Sacrée d'Histria»; A. Avram, I. Bîrzescu, K. Zimmermann, "Die apollinische Trias von Histria».

8. Fouilles K. Zimmermann, 1991 (en cours de publication).

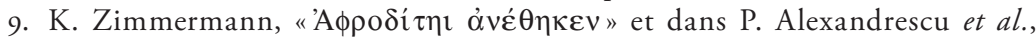
Histria VII, p. 475-476, Ta 319. 
de construction et de reconstruction coïncident en lignes générales avec les grandes étapes de l'évolution de la ville d'Istros. Dans la partie fouillée jusqu'en 1989 de la Zone Sacrée, "trois téménè se partageaient l'espace: celui d'Aphrodite, avec la grande fosse sacrée, celui d'Apollon lètros et celui de Zeus " ${ }^{10}$. Les temples de ces trois divinités portent respectivement les indicatifs $J$, $A$ ' et $A$.

Détruite, d'ailleurs comme toute la ville, vers la fin de l'époque archaïque, la Zone Sacrée fut réaménagée vers le début de l'époque classique selon un programme qui semble avoir continué grosso modo l'héritage archaïque. Les circonstances historiques de la destruction demeurent mal connues. On a songé à l'expédition de Darius de 514 av. J.-C., mais les données archéologiques semblent faire violence à une date aussi haute ${ }^{11}$. Une date aux environs de 490 - serait-ce en liaison avec la campagne de Mardonios en Thrace de 492? - nous semble plus conforme aux témoignages archéologiques. Quoi qu'il en soit de cette hypothèse, il convient de noter que la reconstruction de la Zone Sacrée n'eut lieu qu'à partir des années soixante-dix $\mathrm{du} \mathrm{V}^{\mathrm{e}}$ siècle au plus tôt, si ce n'est que du milieu du même siècle: c'est ce que démontrent les dates attribuées à un dépôt d'amphores de Chios (deuxième quart du $\mathrm{V}^{\mathrm{e}}$ siècle), enfoui en guise d'offrande de fondation sous le sol de la deuxième phase du temple de Zeus ${ }^{12}$, ainsi qu'un complexe révélé par les fouilles plus récentes et dont il sera question plus loin.

La deuxième destruction de la cité, et par conséquent de son téménos, eut lieu sans doute en 313 av. J.-C., à la suite de l'expédition de Lysimaque contre les insurgés du Pont Gauche ${ }^{13}$. La reconstruction de la Zone Sacrée, à partir de la première moitié du III siècle, comporta alors des changements significatifs. Hormis la nouvelle phase (III) du temple d'Aphrodite, dont on conserve même quelques parties de l'élévation, il est notamment question de l'introduction de nouveaux cultes ayant réclamé leur expression architecturale (fig. 2). C'est vers le milieu du III' s. av. J.-C. qu'un nouveau temple fut consacré au "Grand Dieu» (Théos Mégas). Connu dans un premier temps uniquement sur la foi des parties composant sa façade en marbre - dont la dédicace de l'architrave

\footnotetext{
Io. P. Alexandrescu et al., Histria VII, p. 70.

II. Ibid., p. 95-96.

I2. Ibid., p. 99-100; cf. P. Dupont, dans le même volume, p. 242.

I3. Ibid., p. 106-108, qui n'exclut pas pour autant une date plus tardive.
} 
Fig. 2 - La Zone Sacrée à la haute époque hellénistique. 


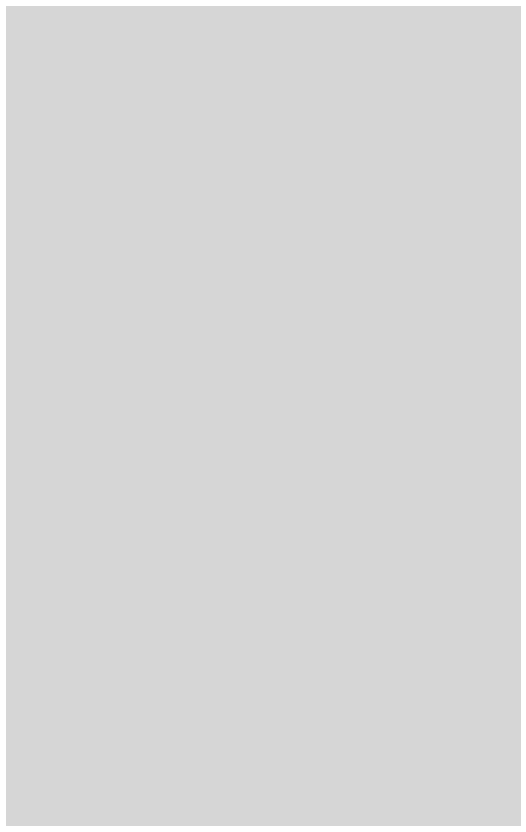

Fig. 3 - Dédicace à Apollon

Pholeutèrios (horos?), ISM I 105.

(ISM I 145) nous fait connaître la divinité tutélaire - l'emplacement de ce temple vient d'être identifié avec les restes du monument $D$. Il s'agit d'un temple orienté vers l'est, dont une bonne partie aurait été détruite, comme dans le cas du temple $A^{\prime}$ (attribué à titre d'hypothèse à Apollon), par le rempart postgothique. Seule la façade était en marbre, le reste de la structure ayant utilisé, comme tous les autres temples, la pierre calcaire taillée ${ }^{14}$.

D'autres cultes supposent des espaces plus modestes qui se laissent parfois percevoir grâce à des inscriptions: c'est le cas d'Apollon Pholeuterios ${ }^{15}$ (fig. 3), ou de Phorkys, une divinité rarement attestée et d'ailleurs encore mal définie, mais dont le culte est révélé à Istros à la fois par une inscription sur pierre et par des graffitis ${ }^{16}$.

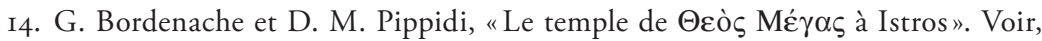
pour les pièces d'architecture et des propositions de reconstitution de la façade, M. Mărgineanu Cârstoiu, "Der Theos-Megas-Tempel von Histria», "Bauelemente des Theos-Megas-Tempels von Histria» et Histria XII, p. 406-431. Pour le rapport entre ces membra disiecta et le monument $D$ et une autre proposition de reconstitution, voir P. Alexandrescu, "Le temple de Théos Mégas redressé» et (avec le concours d'Anişoara Sion), Histria VII, p. 174-186. Pour le caractère de la divinité tutélaire: M. Alexandrescu Vianu, "Théos Mégas» et dans P. Alexandrescu et al., Histria VII, p. 127-137.

I5. ISM I 105 (un horos sacré plutôt qu'une dédicace). Ju. G. Vinogradov, "Heilkundige Eleaten in den Schwarzmeergründungen", rapprochait cette épiclèse du $\phi \omega \lambda \varepsilon \cos _{\varsigma}$ des Eléates et essayait de déceler des traces de la secte fondée par Parménide dans plusieurs cités pontiques. Voir, cependant, à ce propos, les doutes de L. Vecchio, "Medici e medicina ad Elea-Velia», p. 256-257.

16. ISM I 106 (un horos sacré plutôt qu'une dédicace) et deux graffitis commentés en détail par I. Bîrzescu, dans P. Alexandrescu et al., Histria VII, p. 418-420, G 9. 
Ce sont donc ces cultes plus récemment intégrés au panthéon d'Istros qui réclament un nouvel aménagement de l'espace sacré. Et il paraît que l'une des solutions eût été la constitution d'un alignement de monuments de dimensions plutôt modestes, disposés approximativement du nord au sud à partir du monument noté $D$, c'est-à-dire du temple au "Grand Dieu». Ce temple aurait pu constituer un bon repère pour les bases d'inscriptions votives, les anathémata et tous les autres monuments (notés avec des lettres grecques) qui se succèdent vers le sud et qui sont tous plus récents que lui. Néanmoins, à en juger d'après leur position stratigraphique, tous ces monuments ne sont pas parfaitement contemporains ${ }^{17}$.

La troisième destruction de la Zone Sacrée peut être datée des environs de 100 av. J.-C. Il ne s'agirait pas cette fois d'un pillage imputable à un quelconque envahisseur, mais selon toute vraisemblance, d'un tremblement de terre. Le trésor du sanctuaire d'Aphrodite semble avoir été récupéré, et le temple lui-même fut reconstruit visiblement en hâte (phase IV), à l'aide de débris prélevés de ses structures antérieures. Qui plus est, la dernière phase du temple d'Aphrodite (IV: env. 100-48 av. J.-C.) ne présente plus de gradins du côté oriental: un pavage, daté de la première moitié du $\mathrm{I}^{\mathrm{er}} \mathrm{s}$. av. J.-C. et correspondant au dernier niveau de la nouvelle voie sacrée, les a visiblement recouverts et cachés aux yeux ${ }^{18}$. Enfin, la destruction définitive eut lieu vers le milieu du $\mathrm{I}^{\mathrm{er}}$ siècle, fort probablement en 48 av. J.-C., dans le contexte de l'invasion des Gètes de Byrébista ${ }^{19}$. Les traces archéologiques de ce moment dramatique se laissent percevoir de plusieurs manières: couche d'incendie visible sur plusieurs profils, céramiques gètes dans la couche de destruction et - chose plutôt curieuse - même quelques offrandes déposées par les Gètes près du coin SO du temple d'Aphrodite qu'ils venaient d'incendier.

Sans qu'il soit question de nous pencher ici en détail sur ce dossier épineux, nous nous contenterons de rappeler que le secteur fouillé jusqu’à ce jour n'a plus hérité, à l'époque impériale, de son caractère sacré. Dans un premier temps, les restes des temples, des autels et des bases votives

I7. A. Avram, K. Zimmermann, M. Mărgineanu Cârstoiu, I. Bîrzescu, «Nouvelles données sur la Zone Sacrée d'Histria», p. 246.

I8. P. Alexandrescu et al., Histria VII, p. 139-141.

19. P. Alexandrescu, «La destruction d'Istros par les Gètes», Histria VII, p. 142-154 et «La fin de la Zone Sacrée d'époque grecque d'Istros». 
sont soigneusement recouverts d'une couche assez épaisse de terre glaise spécialement apportée d'un autre endroit dans ce but: en effet, on n'y trouve guère de matériels archéologiques. Il s'agit d'une désacralisation de l'endroit, d'un rituel d'abandon de ce lieu, désormais destiné à des activités profanes.

A partir de la fin de l'ère païenne, la place est occupée par quelques ateliers, avant que l'on installe un quartier civil vers la fin du ${ }^{\text {er }}$ s. apr. J.-C. Plus tard, au III ${ }^{\mathrm{e}}$ siècle, le nouveau rempart de la ville (construit après le sac d'Istros par les Goths) détruira, par ses fondations profondes, beaucoup de monuments de l'ancien téménos. Les monuments cultuels d'Istros à l'époque impériale, dont les inscriptions nous confirment l'existence, sont à chercher ailleurs.

Voilà donc l'état de la question au moment où nous avons repris les fouilles en 1990.

\section{Les nouvelles recherches (1990-2009)}

Les principaux problèmes des fouilles reprises en 1990, et dont nous résumons ici les résultats, concernent les débuts du téménos et les monuments situés dans ses parties sud, est et ouest. Les fouilles ont été effectuées, sous la direction de l'Institut d'Archéologie "Vasile Pârvan" de l'Académie Roumaine, par une équipe à laquelle appartiennent, à côté des auteurs de cet article, l'architecte Monica Mărgineanu Cârstoiu (Institut d'Archéologie de Bucarest) et le professeur Konrad Zimmermann (Université de Rostock) ${ }^{20}$.

\section{III.1. La fosse sacrée}

Parmi les principaux acquis des nouvelles fouilles, il convient de mentionner l'identification d'une fosse sacrée. Il s'agit d'une faille profonde, de forme à peu près ovale (longueur max. $14 \mathrm{~m}$, largeur max. $7 \mathrm{~m}$ ), dans le rocher de schiste, à parois abruptes, parfois même quasiment verticales, située juste devant le temple d'Aphrodite (pl. XXXVI). Sa limite nord avait été identifiée pendant les fouilles du début des années

20. Durant ces campagnes, nous avons été aidés par de nombreux assistants et étudiants de Roumanie, d'Allemagne, d'Autriche et des Etats-Unis. 
soixante-dix du siècle passé, lorsque cette faille avait acquis le nom de "grande dépression» dans les carnets de fouilles, mais à l'époque, personne n'avait encore soupçonné son ampleur ${ }^{21}$. Ce n'est qu'entre 1998 et 2004 qu'eut lieu la fouille de cette fosse.

Il va de soi que cette anomalie naturelle aura attiré l'attention des premiers colons milésiens et joué, par conséquent, un rôle important dans l'aménagement du téménos. Il est, d'autre part, évident que cette faille n'a jamais été remplie à l'époque archaïque et au début de l'époque classique, car le matériel plus ancien recueilli jusqu'à ce jour du remplissage ultérieur ne remonte pas, à l'exception de quelques tessons peu significatifs, au-delà de l'extrême fin du $\mathrm{V}^{\mathrm{e}}$ s. av. J.-C. Au vu de cette situation topographique, nous supposons que le temple d'Aphrodite fut orienté en rapport avec cette fosse. Car ce temple présente des degrés à sa façade et du côté est: or, du côté est, le regard tombe sur la fosse et, par conséquent, sur les activités cultuelles que l'on déployait autour d'elle.

$\mathrm{Au}$ fond de la fosse, un enclos rectangulaire repose directement sur le rocher (monument $K$ ). Les parois du rocher ont été, en fait, revêtues de deux murs parallèles, dont les rangées de la base sont conservées. Les rangées sont constituées d'orthostates en calcaire coquillier. Bien en dessus des rangées conservées, on a encore pu identifier des traces d'encastrement de ces murs dans les parois du rocher. Et c'est justement dans l'espace délimité par ces deux murs qu'étaient déposées des amphores, majoritairement thasiennes, dont il nous reste une quantité appréciable de fragments, quelques anses timbrées comprises. Les timbres amphoriques datent ce dépôt de la première moitié du $\mathrm{IV}^{\mathrm{e}} \mathrm{s}$. av. J.-C. ${ }^{22}$. Il est donc très probable, même si pas tout à fait obligatoire, que les murs de l'enclos aient été érigés autour de cette date ${ }^{23}$.

2I. Voir déjà K. Zimmermann, "Ausgrabungen in der Tempelzone von Histria», p. 463 et «Griechische Altäre in der Tempelzone von Histria», p. 148 («... Felsmulde [...], die ursprünglich, d. h. noch vor der Bebauung der Tempelzone möglicherweise als Opfergrube und dann wohl auch dessen kultischer Ausgangspunkt anzusehen ist») et 154 («mögliche Keimzelle des Kultbezirkes»).

22. A. Avram, «Les timbres amphoriques du remplissage de la fosse sacrée du téménos d'Istros», communication faite le 5 février 2010 au Colloque international "Analyse et exploitation des timbres amphoriques grecs», Ecole Française d'Athènes (à paraître dans les actes).

23. Un autre argument pour dater cet enclos du début du IV ${ }^{\mathrm{e}}$ siècle est fourni par l'observation due au géologue Albert Baltres, selon laquelle le calcaire coquillier n'est 
Fig. 4 - Timbre cnidien du «damiurge» Klydoklès, période V, env. 146-108 av. J.-C.

Compte tenu de tous ces éléments, nous estimons qu'il s'agit d'une fosse sacrée, en relation uniquement avec le culte d'Aphrodite ou bien avec plusieurs cultes. Dans une étude publiée il y a quelques années, nous avons avancé plusieurs arguments pour étayer l'hypothèse d'un abaton $^{24}$.

Quoi qu'il en soit, il convient de mentionner que la fosse sacrée cessa son activité quelques dizaines d'années avant la destruction du téménos, suivie par son abandon. Cela se passa vers le début du I ${ }^{\text {er }}$ s. av. J.-C., ce qui est mis en évidence par plusieurs constats.

Tout d'abord, sur tous les profils transversaux utilisés pour le contrôle stratigraphique de la fouille, était visible une couche intermédiaire entre la limite supérieure du remplissage et la trace d'incendie mise en relation avec la destruction de la Zone Sacrée par les Gètes.

Deuxièmement, une rangée de six blocs de grès calcaire taillés gisait directement sur le remplissage de la fosse à son extrémité orientale. Au vu de leur alignement par rapport aux monuments installés à la basse époque hellénistique sur la voie sacrée qui menait au temple du Grand

pas encore attesté dans les monuments d'Istros avant la fin du Ve s. - début du IVe $s$. av. J.-C.

24. A. Avram, K. Zimmermann, M. Mărgineanu Cârstoiu, I. Bîrzescu, «Nouvelles données sur la Zone Sacrée d'Histria», p. 243-245. Deux graffitis d'Olbia mentionnent justement «les abata d'Aphrodite» (IGDOP 71 a et b). On a, par conséquent, la preuve que de tels abata existaient à Olbia, dont on connaît par ailleurs les similitudes cultuelles avec Istros. 
Dieu, et dont il a été question plus haut, ces blocs auraient pu constituer une bordure de cette voie. Quelle qu'eût été leur destination, c'est surtout leur position stratigraphique qui est à retenir: on a donc affaire à un aménagement intermédiaire entre le remplissage de la fosse et la destruction définitive de la Zone Sacrée.

Troisièmement, et dernièrement, le matériel provenant du remplissage s'échelonne du IV ${ }^{\mathrm{e}}$ siècle jusque vers le début du I ${ }^{\mathrm{er}}$ s. av. J.-C. Les exemplaires les plus récents pourraient être quelques timbres rhodiens et cnidiens (fig. 4) de la "période V" (avant env. 108 av. J.-C.), ainsi que deux fragments de céramique à décor en relief du type "Hadra», largement datés de la deuxième moitié du $\mathrm{II}^{\mathrm{e}}$ siècle et de la première moitié du $\mathrm{I}^{\mathrm{er}} \mathrm{s}$. av. J.-C.

Tout bien considéré, il reste à s'interroger sur la date et les circonstances du remplissage intentionnel de la fosse. La chronologie du temple d'Aphrodite, brièvement présentée plus haut, semble en fournir la clé. Nous avons déjà mentionné que le temple fut détruit vers $100 \mathrm{av}$. J.-C., semble-t-il, par un tremblement de terre. Ce qui est maintenant confirmé par nos fouilles: l'inclinaison visible du mur sud de l'enclos situé dans la faille devant le côté est du temple d'Aphrodite pourrait, en effet, s'avérer tout à fait conforme aux conséquences d'une secousse tellurique. D'autre part, l'érosion du rocher aurait pu menacer de faire s'écrouler le temple même (voir l'avancement de la limite actuelle de la faille vers l'ouest, ayant d'ailleurs entraîné la chute de quelques marches de la plate-forme du temple). Il convient de rappeler que lors de la dernière reconstruction du temple d'Aphrodite, les marches du côté est furent recouvertes d'un pavage. Disparition des gradins et remplissage de la fosse vont donc de pair, ce qui s'explique d'ailleurs aisément: pour autant qu'il n'existât plus de fosse (autant dire que l'on abandonnât sa fonction rituelle), il n'était plus besoin de gradins du côté est du temple. Ce qui nous semble être une explication raisonnable pour le remplissage de la fosse. 
Fig. 5 - La Zone Sacrée vers la fin du VII e siècle et dans la première moitié du VIe s. av. J.-C. Plan général.

III.2. Nouveaux monuments et nouveaux problèmes

a) La première phase de l'époque archaïque: jusque vers le milieu du $\mathrm{VI}^{\mathrm{e}}$ siècle av. J.-C.

L'un des objectifs majeurs des recherches des dernières années concerne les débuts de la Zone Sacrée. Des traces plutôt discrètes d'aménagements du premier siècle d'existence du téménos furent découvertes par les fouilles plus anciennes, sans qu'il soit pour autant possible d'aboutir à une image plus claire de cet ensemble avant le milieu du $\mathrm{VI}^{\mathrm{e}}$ siècle (fig. 5). Les premiers aménagements furent installés directement sur le rocher de schiste vert, dont la surface avait été nivelée à l'aide d'une couche de terre brune. Nous ajoutons qu'il n'y a aucun indice d'activité humaine à la veille de l'arrivée des Milésiens. 


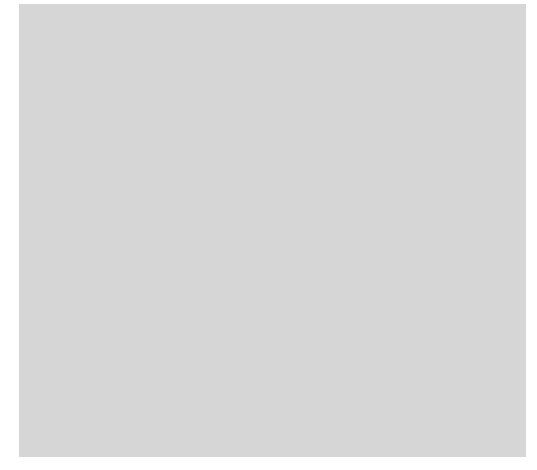

Fig. 6 - Vase de cuisine éginète.

Seule la grande faille, déjà mentionnée, se présentait dans sa splendeur aux yeux des colons. Elle aura sans aucun doute joué un rôle fondamental dans le choix de l'emplacement des sanctuaires et dans l'aménagement de l'espace sacré.

Un premier aménagement du rocher se laisse percevoir au sud de cette fosse. Il s'agit d'une cavité de forme circulaire, assez superficielle (env. 10-20 cm de profondeur), de surface lisse et d'un diamètre de $75 \mathrm{~cm}$. Près de cette cavité se trouvent, à quelques centimètres vers le NE, deux petits trous creusés dans le rocher, dans lesquels étaient probablement fixés des palis.

Une deuxième cavité dans le rocher $(16 \mathrm{~cm}$ de profondeur) se trouve plus à l'est. Elle est, par contre, de forme rectangulaire et présente une surface irrégulière. Seule une surface de $2,70 \mathrm{~m}$ x $1,70 \mathrm{~m}$ a pu être recherchée, car ses côtés est et sud se trouvent sous les soubassements des murs d'époque romaine. Cette cavité coupe la couche brune déposée sur le rocher, de laquelle ont été récupérés plusieurs fragments céramiques datables de la fin du VII ${ }^{\mathrm{e}}$ siècle et du tout début du VI ${ }^{\mathrm{e}}$ siècle: coupes à oiseaux tardives, céramiques de cuisine tournées ${ }^{25}$ (fig. 6), amphores milésiennes, clazoméniennes, éoliennes, attiques du type SOS, chiotes et samiennes.

Une troisième cavité, cette fois de forme ovale, a été identifiée vers la limite sud de la fouille actuelle (env. $3 \mathrm{~m}$ EO et 1,65 m NS). Il est pour autant difficile de se prononcer sur la destination de ces cavités pratiquées dans le rocher.

A l'exception des fondations de l'ancien oikos d'Aphrodite, lequel date de la première moitié du $\mathrm{VI}^{\mathrm{e}}$ siècle ${ }^{26}$, il n'y a pour l'instant aucune autre trace de construction ayant utilisé la pierre. En revanche, à côté des cavités dans le rocher que nous avons présentées, il convient d'attirer l'attention sur les traces de deux aménagements identifiés vers le coin SE

25. Dont un fragment à attribuer à une production éginète (observation due au $\mathrm{D}^{\mathrm{r}}$ Gudrun Klebinder-Gauss).

26. P. Alexandrescu et al., Histria VII, p. 68-70. 
de notre fouille, de dimensions de $1,10 \mathrm{~m} \times 1,17 \mathrm{~m}$ et $1,46 \mathrm{~m} \times 0,85 \mathrm{~m}$ respectivement.

Du point de vue quantitatif et qualitatif, le mobilier votif que l'on a récupéré à proximité de ces complexes ne s'éloigne pas trop de l'image d'ensemble révélée par le téménos dans sa phase initiale. Il convient de retenir quelques terres cuites, dont un vase anthropomorphe (adolescent nu) portant une dédicace quasiment illisible gravée sur sa partie postérieure, quelques fragments de faïence et de millefiori.

b) L'époque archaïque tardive: du milieu du VI ${ }^{\mathrm{e}}$ siècle au début du $\mathrm{V}^{\mathrm{e}}$ s. av. J.-C.

De la description fournie jusqu'ici, rien n’aurait laissé présager l'essor que prit la Zone Sacrée peu après le milieu du VI e siècle, avec ses trois temples en pierre (Zeus, Aphrodite et peut-être Apollon), sans préjudice d'autres monuments sacrés de moindre envergure. Les causes de ce développement extraordinaire ont été mises en évidence ailleurs ${ }^{27}$, ce qui nous épargne la peine d'y insister. Hormis plusieurs précisions ayant trait à des monuments déjà connus, les nouvelles fouilles en ont ajouté d'autres, que nous nous proposons de présenter brièvement.

Les fouilles ont été dirigées surtout vers le sud et vers l'ouest. Rappelons que la limite est de la Zone Sacrée se trouve maintenant sous les eaux limoneuses du lac de Sinoé, et dont il n'est pas encore question d'étudier les traces, et que la limite nord est communément considérée comme étant indiquée par le tronçon nord de l'enceinte d'époque hellénistique, bien qu'à l'époque archaïque, une extension du téménos même au-delà de ce point ne soit pas, pour le moins théoriquement, à exclure.

La limite ouest pourrait être indiquée par le monument $C$, interprété comme propylon ${ }^{28}$. Dans ce secteur, les fouilles récentes se sont attaquées à l'espace situé à l'ouest du temple d'Aphrodite.

27. Voir surtout P. Alexandrescu, «Histria in archaischer Zeit», p. 56-62 = L'Aigle et le dauphin, p. 85-95.

28. P. Alexandrescu (avec le concours d'Anişoara Sion), Histria VII, p. 187-197. Selon le modèle proposé par M. Mărgineanu Cârstoiu, «Plans de villes romaines en Mésie inférieure», p. 307 et fig. 5, une rue identifiée dans le quartier civil (sur le "plateau») menait justement au monument $C$, ce qui fournirait un atout majeur à son interprétation comme entrée monumentale dans la Zone Sacrée. En revanche, l'appartenance de l'architrave portant l'inscription ISM I 144 au monument $C$, proposée 
Un premier complexe digne d'attirer l'attention est constitué par un amas de décombres de tuiles découvert à un mètre de la plate-forme du temple d'Aphrodite, au niveau de son naos, sur une surface d'environ $1,50 \mathrm{~m} \times 2 \mathrm{~m}$. Les types auxquels appartiennent ces tuiles indiquent que nous avons affaire sans l'ombre d'un doute à une partie du toit effondré du temple archaïque d'Aphrodite détruit à la fin de l'époque archaïque (pl. XXXVII). Des parties du même toit avaient été découvertes lors des fouilles des années soixante-dix du siècle passé dans le coin NO du temple et surtout à l'intérieur, dans son pronaos ${ }^{29}$.

Les dernières découvertes (2007 et 2008) ont, par contre, contribué à une meilleure connaissance de la date et des circonstances de la destruction du temple à la suite d'un incendie, et cela d'autant plus que ces tuiles n'ont plus été dérangées après leur chute: elles reflètent le moment même de l'effondrement du toit. A preuve, l'alignement constitué de cinq plaques de couverture, retrouvées avec des poutres carbonisées et des morceaux de plomb destinés à l'attache, ainsi que des tuiles courantes chevauchées par des couvre-joints. Tous ces objets étaient tombés sur une surface de terre glaise, représentant le sol extérieur du temple au moment de sa destruction et qui, au contact avec les poutres brûlées, a pris une couleur rougeâtre. Vers l'extrémité de cet amas, à environ 2,80 m de la plate-forme du temple, se trouvaient des fragments appartenant à un chapiteau ionique en grès calcaire jaune et de nombreuses antéfixes peintes (pl. XXXVIII.1). Le chapiteau ionique se trouve apparemment en position secondaire: appartenant à la façade du temple, il aura été déplacé de l'endroit de sa chute et abandonné sur l'amas de tuiles formé à la suite de l'effondrement du toit. S'ajoute un fragment de sima que l'on peut maintenant reconstituer à l'aide des trouvailles plus anciennes. L'analyse de quelques fragments de poutres a mis en évidence deux essences, le frêne et le chêne ${ }^{30}$.

Au nord de ce complexe, un puits datant de la basse époque romaine, déjà identifié par les recherches des années cinquante et fouillé il y a

par P. Alexandrescu et A. Sion (p. 195-197), est rejetée par M. Mărgineanu Cârstoiu, Histria XII, p. 455-465: elle pense plutôt au temple même d'Apollon, lequel aurait été refait a fundamentis à la fin du IVe s. av. J.-C.

29. K. Zimmermann, "Zu den Dachterrakotten griechischer Zeit aus Histria», "Archaische Dachterrakotten aus Histria» et dans Histria VII, p. 463-485.

30. Analyse réalisée par le professeur Karl-Uwe Heußner de l'Institut archéologique allemand de Berlin. 
deux ans de manière exhaustive, a dérangé toute la stratigraphie jusqu'au rocher. En revanche, au sud et au nord de ce puits, la stratigraphie s'est avérée complète. La remarque la plus intéressante concerne la fondation d'une muraille orientée approximativement NS, se dirigeant presque parallèlement à la plate-forme du temple d'Aphrodite et identifiée jusqu'ici sur une longueur de plus de $19 \mathrm{~m}$. Elle présente une largeur de $1 \mathrm{~m}$ à $1,10 \mathrm{~m}$ et est composée de grandes dalles de schiste et de calcaire de forme irrégulière. Cette fondation de muraille est surmontée par le monument $C$ (fig. 7). C'est à l'est de ce monument que l'on a également trouvé les seuls restes de son élévation: deux petits blocs en grès calcaire. Si cette succession stratigraphique n'a rien de surprenant (rappelons que le monument $C$ date de l'époque hellénistique), il est en revanche extrêmement important de constater que la surface de terre glaise sur laquelle sont tombées les tuiles dont il vient d'être question et qui nous indique, par conséquent, le niveau du sol extérieur du temple archaïque au moment de sa destruction, recouvre visiblement la fondation de cette muraille. Cette dernière est donc plus ancienne que le temple en pierre et elle aurait pu correspondre à l'oikos ayant précédé cette phase, soit à la première moitié $\mathrm{du} \mathrm{VI}^{\mathrm{e}}$ siècle. Le seul élément de datation absolue que nous pouvons avancer pour l'instant est constitué par plusieurs fragments appartenant à une coupe de la classe Siana du peintre "Heidelberg", datée peu avant le milieu du VI ${ }^{\mathrm{e}}$ siècle. Désaffectée fort probablement lors de la construction du temple en grès calcaire d'Aphrodite peu après le milieu du VI ${ }^{\mathrm{e}}$ siècle, cette muraille, qui ne présente pas, sur la longueur dégagée jusqu'à l'heure qu'il est, des ramifications dans quelque direction que ce soit, peut laisser la porte ouverte à plusieurs hypothèses. On n'en retiendra que deux, car plus vraisemblables: soit un péribole datant de la première phase du téménos (c.-à-d. avant les constructions en pierre), soit un premier essai, finalement abandonné, de jeter le fondement d'une plate-forme pour le temple d'Aphrodite.

Toujours à proximité du monument $C$, les anciennes fouilles étaient tombées sur un enclos semi-circulaire composé de plaques de chant de grès calcaire jaunâtre, épaisses d'environ $15 \mathrm{~cm}$. Il est évident qu'un tel aménagement ne supportait aucune élévation et que, par conséquent, il jouait plutôt le rôle d'une démarcation. La seule chose que l'on puisse dire pour l'instant est que cet aménagement était antérieur au 
Fig. 7 - A l'ouest du temple d'Aphrodite, fondation d'une muraille archaïque surmontée par le monument $C$ (à gauche). A droite, aménagement semi-circulaire d'époque classique. Au fond, murs d'époque romaine. 
monument $C$ : il date, par conséquent, soit du bas archaïsme, soit du haut classicisme.

Plusieurs fragments architectoniques furent découverts dans le même secteur hors contexte, soit en tant que débris réutilisés dans la structure des murs de basse époque romaine, soit égarés dans diverses

Fig. 8 - Autel en miniature découvert couches posthellénistiques. près du temple d'Aphrodite.

Quant aux trouvailles à caractère votif, elles deviennent manifestement plus nombreuses. Près du temple d'Aphrodite furent découvertes des terres cuites appartenant à des types semblables aux pièces déjà connues grâce aux fouilles plus anciennes, à savoir des femmes assises ou debout, la tête voilée, ou bien un pansu conservant des traces de peinture rouge. On en reconnaît l'empreinte milésienne. S'ajoute une arula à marches en plâtre découverte en 2008 (fig. 8). Une autre catégorie de mieux en mieux représentée est constituée par les graffitis porteurs de dédicaces, recueillis notamment au sud et à l'est de la fosse sacrée: une dédicace à Apollon sur une coupe attique à yeux ${ }^{31}$, une autre à Dionysos à l'intérieur d'une cruche Fikellura, et enfin une autre à Hermès sur une coupe attique de la classe des Petits Maîtres ${ }^{32}$.

La plus importante découverte des dernières années concerne sans aucun doute les restes d'un monument identifié en 2007 vers le coin SE de la fouille actuelle, tout près de l'enceinte postgothique et sous les soubassements d'une basilique chrétienne du $\mathrm{V}^{\mathrm{e}}$ s. apr. J.-C. entièrement dégagée en 1997.

Il s'agit d'un nouveau monument cultuel - noté $M$ - de dimensions plutôt modestes et orienté approximativement NS, comme tous les autres temples archaïques connus jusqu'à ce jour. Les fondations de cet édifice, reposant directement sur le rocher, sont plutôt bien conservées, mais

3I. A. Avram, I. Bîrzescu, K. Zimmermann, «Die apollinische Trias von Histria», p. $117, n^{\circ} 25$ et fig. 6 a-c.

32. I. Bîrzescu, "Some Ceramic Inscriptions from Istrian Sanctuaries», p. 171-172 et «Histria», p. 208 et fig. 1/7. 
surmontées en grande partie par les soubassements de la future basilique, ce qui empêche une recherche exhaustive (pl. XXXVIII.2). Le plan de cet édifice a pu être reconstitué en partie grâce à plusieurs coupes pratiquées surtout ces dernières années à l'intérieur de la basilique. Deux pièces peuvent d'ores et déjà être documentées du point de vue archéologique, alors que pour une troisième, nous ne disposons que de quelques faibles indices. A la suite des décapages qui se sont succédé depuis la fin de l'Antiquité - ne fût-ce qu'à penser à la construction du rempart et, plus tard, de la basilique - et surtout pendant le bas Moyen Age, à une époque où le rempart postgothique était devenu une véritable carrière de pierre pour la forteresse ottomane de Vadu (Caraharman), située à $10 \mathrm{~km}$ à vol d'oiseau, les couches grecques ont été presque entièrement enlevées.

Il y a pour autant quelques données que l'on pourrait considérer comme déjà acquises. L'édifice comporte une longueur d'environ $11 \mathrm{~m}$ et une largeur d'environ $6,30 \mathrm{~m}$. La largeur de ses fondations est de $67-70 \mathrm{~cm}$. A une seule exception près, les murs sont surmontés par les soubassements de la basilique. La stratigraphie de la surface fouillée a révélé trois niveaux archaïques et un niveau classique. Un premier niveau, qui ne dépasse pas le milieu du $\mathrm{VI}^{\mathrm{e}}$ siècle, est mis en évidence par le nivellement du rocher au moyen d'une couche d'argile glaise comportant aussi deux fosses et un foyer. L'édifice de culte appartient à un deuxième niveau, caractérisé par un tassement dans la composition duquel l'on a trouvé entre autres quelques fragments d'amphores de Chios du type Lambrino A2 datables d'environ 540-510/500 av. J.-C. Quelques réparations semblent avoir eu lieu durant le dernier quart du même siècle. Plusieurs pointes de flèches à valeur monétaire ont été trouvées sous le sol de la pièce nord de l'édifice. Le troisième niveau, correspondant à la deuxième phase de l'existence du monument, date des environs de 500. Le mur intérieur médian révèle des traces de réparation.

Contrairement à tout ce que l'on connaissait des autres édifices de la Zone Sacrée, les fondations de ce monument sont constituées majoritairement de fragments informes de marbre, auxquels s'ajoutent dans une moindre mesure des blocs de grès calcaire. Il n'y avait jusqu'ici aucun indice sur l'utilisation du marbre dans les édifices sacrés d'époque archaïque à Istros. Aussi faut-il rappeler que le marbre était un matériau procuré uniquement par importation.

L'édifice $M$ fut détruit par un incendie. La couche de destruction, bien définie du point de vue archéologique, contenait de nombreux fragments 
de briques crues conservant des traces de l'action du feu, parfois à surfaces lisses - ce qui montre qu'elles avaient appartenu aux parois de l'édifice -, des restes carbonisés en provenance de poutres, dont les analyses ont mis en évidence des essences telles le chêne et le pin, et surtout une quantité appréciable de tuiles. Des morceaux de briques crues tombées ont souvent été découverts in situ, sur le niveau de terre glaise, lequel conservait à son tour de fortes traces d'incendie. Ce niveau est d'ailleurs bien conservé sur toute la surface recherchée. Sous ce niveau, il y avait une couche distincte de terre ameublie de couleur gris-brun, mêlée à de petites pierres, des coquilles, des os et des céramiques datables de la fin $\mathrm{du} \mathrm{VI}^{\mathrm{e}}$ siècle. Ceci étant, l'on a affaire à un édifice érigé dans le troisième tiers, ou peut-être à l'extrême fin du $\mathrm{VI}^{\mathrm{e}}$ siècle, et détruit, semble-t-il, quelques décennies après, selon toute vraisemblance, au même moment que les autres monuments sacrés de la zone. L'étude détaillée du mobilier pourra sans doute fournir des précisions supplémentaires sur la date et les circonstances de cet événement.

Une bonne partie de la toiture a apparemment échappé au feu, car, après la destruction, plusieurs tuiles ont été disséminées au sud du même édifice et mêlées à des fragments céramiques, notamment d'amphores. A défaut de se fier à la chronologie encore assez fluctuante des tuiles, il vaut mieux compter sur les fragments d'amphores de Chios à col renflé et sans peinture sur la lèvre et d'amphores thasiennes du début de l'époque classique: ce qui ferait dater notre complexe du deuxième quart $\mathrm{du} \mathrm{V}^{\mathrm{e}}$ siècle, une date qui correspondrait d'ailleurs grosso modo aux travaux de restauration accomplis aux temples de Zeus et d'Aphrodite ${ }^{33}$. Les tuiles disséminées dans ce "pavage céramique" sont du même type que celles que l'on a prélevées de la couche d'incendie: il n'y a que les traces de brûlure secondaire et le degré de fragmentation qui en font la différence. Durant les trois dernières campagnes, on a recueilli quelques centaines de fragments. Il s'agit majoritairement de tuiles courantes et de couvre-joints, d'une forme qui diffère du type déjà établi pour la toiture du temple d'Aphrodite. S'ajoutent dans une moindre mesure des antéfixes, des simas et des tuiles d'égout, le tout étant caractérisé par une polychromie extraordinaire, avec une préférence pour le blanc et le rouge (pl. XXXIX.1).

33. P. Alexandrescu et al., Histria VII, p. 98-104. 
Le mobilier de ce petit édifice, lequel a toutes les chances d'être considéré comme un naiskos, a pu être récupéré en partie de la couche de destruction. Parmi les vases cultuels, il convient de mentionner en premier un périrrhantèrion céramique décoré avec des frises à oves, dont on conserve le bassin et le support. Le mobilier votif est complété par des protomes en terre cuite, féminins pour la plupart, dont quelques-uns conservent en partie la polychromie d'origine (pl. XL). Ils appartiennent tous au type milésien et sont datables des environs de 500 av. J.-C. Une autre terre cuite, laquelle semble avoir été accrochée à une paroi de briques crues, représente un coq peint en cinq couleurs ${ }^{34}$ (pl. XXXIX.2). A ce même inventaire appartenaient aussi des pendentifs et des feuilles d'or à décor à palmettes qui ornaient sans doute une statue cultuelle d'ivoire. En effet, les quelques fragments d'ivoire que l'on a également trouvés semblent conforter une telle hypothèse. Pour leur part, les feuilles d'or ont fait l'objet d'analyses ${ }^{35}$ et ont été évaluées à 22 et 23 carats respectivement. Si le nombre de fragments de vases céramiques de cette couche est plutôt insignifiant, il convient, par contre, de ne pas passer sous silence un fragment d'inscription archaïque sur pierre, une dédicace faite par un certain Héphaistodôros: il s'agit de la quatrième découverte de ce genre à Istros et de la troisième dans le téménos.

Vaut ce que vaut le caractère éminemment féminin des terres cuites, il serait peut-être permis de supposer un petit temple consacré à une déesse, sans doute la Mère des Dieux ${ }^{36}$. Néanmoins, parmi les graffitis trouvés durant les campagnes précédentes, il n'y en a aucun qui puisse venir à l'appui d'une telle hypothèse.

c) L'époque classique

Contrairement aux temples d'Aphrodite et de Zeus, restaurés après la catastrophe dans le courant de la première moitié du $\mathrm{V}^{\mathrm{e}}$ siècle, le monument $M$ semble avoir été définitivement abandonné après sa destruction. Il faut attendre l'époque hellénistique pour voir de nouveaux édifices de

34. Bonne analogie à Panticapée: P. F. Silant'eva, Terrakoty Pantikapeja, p. 16, pl. 3.4.

35. Grâce à l'obligeance de M. Bogdan Constantinescu de l'Institut de physique atomique de Bucarest.

36. Voir, à Olbia, A.S. Rusjaeva et al., Drevnejšij temenos Ol'vii Pontijskoj, p. 20-22. 
culte renaître à cet endroit, signe d'un nouvel aménagement dont il sera question ci-dessous.

Au sud du temple d'Aphrodite, les monuments d'époque classique font entièrement défaut. Les habitations d'époque romaine pourraient en être largement responsables, car on a constaté à plusieurs reprises que leurs fondations coupent la stratigraphie jusqu'aux couches archaïques, voire jusqu'au rocher.

L'aménagement semi-circulaire situé à l'ouest du temple d'Aphrodite pourrait appartenir à cette époque, mais sur ce point il faut encore attendre des précisions stratigraphiques. Le fragment d'une plaque monumentale de marbre, conservant les restes d'une inscription dont le texte avait été disposé en stoichèdon, suggère qu'il y avait pourtant des rebondissements dans l'activité de construction. Rappelons que pendant la première moitié du $\mathrm{IV}^{\mathrm{e}}$ siècle a été installé dans la fosse sacrée l'enclos que nous avons mentionné à un autre end roit de notre exposé.

d) La haute époque hellénistique

La destruction d'Istros en 313 av. J.-C., imputable, on l'a vu, à Lysimaque, a été suivie dans le téménos par un nouvel essor constructif que l'on daterait plutôt de la première moitié, voire du deuxième quart du III ${ }^{\mathrm{e}}$ siècle ${ }^{37}$.

Le principal monument que l'on ajoute à cette époque est le temple de Théos Mégas ${ }^{38}$. Vu son emplacement, il est fort probable qu'une deuxième

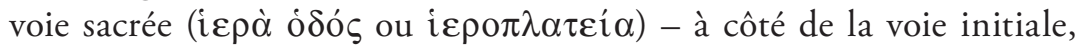
laquelle passait par le monument $C$, interprété comme propylon - se dirigeait vers ce nouveau temple. La preuve serait fournie par les monuments votifs alignés du sud vers le nord jusqu’à proximité du temple du «Grand Dieu» et dont la plupart ont été découverts lors des anciennes fouilles.

Cette image est confortée par nos recherches, qui ont mis en évidence l'existence d'une voie aménagée au III siècle et refaite ensuite à deux reprises, au $\mathrm{II}^{\mathrm{e}}$ siècle et dans la première moitié du I ${ }^{\mathrm{er}} \mathrm{s}$. av. J.-C. Elle se présente comme un gravier de débris calcaires mélangé à de la terre glaise et ayant dans sa composition des tessons. Parmi les tessons constituant la structure de ce "pavage", on compte entre autres quelques timbres amphoriques capables de suggérer des datations plus précises.

37. P. Alexandrescu et al., Histria VII, p. 109-127.

38. Voir supra, note 14. 
C'est sur cette voie que l'on a découvert quatre nouvelles bases, dont trois in situ, à l'est de la fosse sacrée. Rappelons que, contrairement à ses fondations, le sol de la basilique dégagée en 1997 n'a pas dérangé les couches d'époque hellénistique, ce qui nous a permis d'en établir une stratigraphie plutôt cohérente. Certes, il y aurait eu, dans ce même secteur, plusieurs monuments de ce genre, sauf que les décapages successifs en auront effacé les traces. A en juger d'après leur position stratigraphique, les trois bases in situ appartiennent au deuxième niveau hellénistique, soit au II siècle. Comme plusieurs autres bases de la même voie, découvertes lors des fouilles plus anciennes, elles réutilisent des débris récupérés de monuments abandonnés. Compte tenu de la dispersion des trouvailles, il est pratiquement impossible de remettre à leur place les différentes stèles évoquant des divinités et trouvées en position secondaire à peu près partout. Aussi nous accordera-t-on que certains horoi sacrés, dont ceux d'Apollon Pholeutèrios (ISM I 105) ou bien de Phorkys (ISM I 106), dont il a été question plus haut, aient été imbriqués sur des bases dont nos alignements nous offrent un nombre suffisant d'exemples.

Tout bien considéré, l'hypothèse d'une deuxième voie sacrée aménagée à la haute époque hellénistique, soigneusement entretenue et refaite à plusieurs reprises, nous semble plutôt recevable. Si tel est le cas, un propylon secondaire, comme point initial de cette nouvelle voie sacrée, serait à attendre plus au sud, dans une zone qui n'a pas encore été touchée par les fouilles.

A l'extrémité opposée de cette voie sacrée, tout près du monument $C$ censé être le propylon d'époque hellénistique, on est maintenant en train de dégager un mur composé de blocs en grès calcaire soigneusement taillés et qui a ensuite servi de soubassement pour les maisons de l'îlot romain. $\mathrm{Vu}$ son aspect, sa relation avec le monument $C$, sa position stratigraphique lui assurant une datation au III siècle, il pourrait s'agir d'un péribole érigé à la limite ouest de la zone en même temps que le monument $C$. Néanmoins, la fouille étant en cours et les données accumulées jusqu'à ce jour encore incomplètes, il est prématuré d'en dire davantage.

Hormis tous ces monuments découverts in situ, il faut une fois de plus se pencher sur les débris de pièces d'architecture trouvés en position secondaire, soit réutilisés dans les maisons de l'insula installée à l'époque impériale, soit tout simplement abandonnés à divers endroits. Ces membra disiecta suggèrent que, contrairement aux époques précédentes, largement dominées par l'ordre ionique, l'époque hellénistique a 
Fig. 9 - Frise en marbre à lions et griffons.

l'air d'appartenir plutôt à l'ordre dorique ${ }^{39}$. Outre le temple du «Grand Dieu ", dont on a d'ailleurs trouvé en 2009 un nouveau fragment de corniche à mutules, qui conserve aussi des traces de couleur rouge, il suffit de faire état de plusieurs frises en calcaire à métopes et triglyphes ou de quelques fragments de chapiteaux. D'autres frises, l'une en calcaire et décorée de bucranes et de guirlandes, proche du point de vue stylistique de la base de la statue de culte d'Aphrodite ${ }^{40}$, l'autre en marbre et à la représentation de lions et de griffons (fig. 9), ornaient des monuments de moindres dimensions. Quant au mobilier votif, il est tout aussi représentatif que pendant les époques antérieures.

\section{e) La basse époque hellénistique}

L'image de la Zone Sacrée change manifestement après environ $100 \mathrm{av}$. J.-C. Désormais on n'aura plus affaire qu'à de petits changements et à une série de réparations ponctuelles plus ou moins inspirées.

A l'occasion des fouilles faites dans les années 1990 au sud du temple d'Aphrodite, nous avons identifié un nouveau monument (noté $L$ ), qui, dans l'état actuel, est très mal conservé: on n'en dispose que de la moitié. Il date, selon sa position stratigraphique, de la première moitié du

39. Hormis le temple de Théos Mégas et le monument auquel aurait appartenu le bloc d'architrave porteur de l'inscription ISM I 144 (cf. note 28), M. Mărgineanu Cârstoiu parvint à reconstituer, sur la foi de plusieurs éléments, deux autres édifices cultuels doriques d'époque hellénistique, dont on n'a pas encore identifié l'emplacement: le temple «X» (Histria XII, p. 432-454 et pl. CXXXVII-CXL) et le temple (?) «N» (ibid., p. 465-467). Cf. ibid., p. 458, note 1245: «il n'y a aucune preuve de la pénétration du dorique dans l'architecture histrienne pendant la période archaïque ou classique».

40. M. Alexandrescu Vianu, Histria IX, p. 89-96, no 105 et pl. 44-45; M. Mărgineanu Cârstoiu, Histria XII, p. 397-406 et pl. CXXIII-CXXIV. 
$\mathrm{I}^{\text {er }}$ s. av. J.-C. et se compose uniquement de blocs récupérés d'autres monuments de la zone, y compris, semble-t-il, du temple d'Aphrodite même. Selon toute probabilité, vu son emplacement juste en face de ce dernier, il s'agirait de l'autel d'Aphrodite correspondant à la dernière phase du temple. On ignore l'emplacement de l'autel originaire : celui-là semble avoir été démantelé après une destruction massive, peut-être toujours à la suite du supposé tremblement de terre dont il a déjà été question. Une telle hypothèse trouverait un appui indirect dans la situation constatée au monument $C$ (propylon). Là, on a affaire à un monument hellénistique et on suppose qu'un prédécesseur archaïque a été démantelé à un certain moment, pour céder la place à une nouvelle construction $a$ fundamentis. Cela pourrait suggérer que de telles pratiques étaient bien usuelles lors des différents réaménagements de l'espace sacré.

L'existence d'un autre autel, situé à quelques mètres plus au sud du monument $L$, pourrait être suggérée par les restes d'une construction presque entièrement détruite par une cour pavée avec des dalles de schiste et appartenant à l'insula d'époque romaine.

Enfin, vers la limite sud de notre fouille actuelle, un long mur composé, lui aussi, de blocs récupérés de monuments plus anciens et, à son tour, réutilisé comme soubassement d'un mur de l'îlot romain pourrait être interprété, à titre d'hypothèse, comme un péribole marquant la limite sud de l'espace sacré à la basse époque hellénistique (après env. 100 av. J.-C.). Comme pour le monument $L$ (autel supposé d'Aphrodite pendant la dernière période), ou comme pour la dernière phase du temple d'Aphrodite (à vrai dire, une réparation plutôt qu'une restauration selon un programme cohérent), il s'agissait probablement d'une mesure conçue comme provisoire. Même au cas où la fonction de péribole de cette muraille serait confirmée par les fouilles à venir, il est peu probable que la Zone Sacrée s'arrêtait là à l'époque archaïque. A ses débuts, elle aurait dû être beaucoup plus étendue vers le sud.

L'image générale qui résulte de toute cette période datée d'environ 100-48 av. J.-C. est plutôt décevante: réparation hâtive du temple d'Aphrodite, comportant entre autres l'abandon de ses degrés du côté est, disparition de la fosse sacrée, sans doute réfection tout aussi superficielle de l'autel d'Aphrodite, en réutilisant massivement des décombres et seules quelques petites nouvelles bases sur le troisième et dernier niveau de la voie sacrée menant vers le temple du "Grand Dieu». Il est donc permis de constater que, si le téménos vit sa fin lors du sac des Gètes vers 
48 av. J.-C., l'ensemble des monuments qui se présentaient aux yeux des fidèles après la destruction due sans doute à un tremblement de terre aux environs de 100 av. J.-C. ne constituait plus qu'un piètre souvenir de ce que la Zone Sacrée avait été naguère.

Alexandru Avram

Université du Maine, Le Mans

Iulian BîRzEscu

Institut d’Archéologie «Vasile Pârvan », Bucarest 


\section{BIBLIOGRAPHIE}

\section{Abréviations}

ISM I = PIPPIDI, Dionisie Mihai, Inscripţiile din Scythia Minor grecești şi latine I. Histria şi împrejurimile, Bucureşti, Editura Academiei Române, 1983.

$I G D O P=$ DuboIs, Laurent, Inscriptions grecques dialectales d'Olbia du Pont, Genève, Droz, 1996 (Hautes études du monde grécoromain 22).

\section{Etudes}

Alexandrescu, Petre, "Notes de topographie histrienne», Dacia N. S., 22 (1978), p. 331-342.

—, "Histria in archaischer Zeit", in Histria. Eine Griechenstadt an der rumänischen Schwarzmeerküste, hrsg. von Petre Alexandrescu, Wolfgang Schuller, Konstanz am Bodensee, Universitätsverlag, 1990, p. 47-101 (Konstanzer althistorische Vorträge und Forschungen 25).

—, "La destruction d'Istros par les Gètes. 1. Dossier archéologique », Il Mar Nero, 1 (1994), p. 179-214.

—, L'Aigle et le dauphin. Etudes d'archéologie pontique, Bucarest/Paris, Editura Enciclopedică/De Boccard, 1999.

—, "Le temple de Théos Mégas redressé», Dacia N. S., 43-45 (19992001), p. 79-96.

—, "La fin de la Zone Sacrée d'époque grecque d'Istros", Dacia N. S., 51 (2007), p. 211-219.

Alexandrescu, Petre, Schuller, Wolfgang (Hrsg.), Histria. Eine Griechenstadt an der rumänischen Schwarzmeerküste, Konstanz am Bodensee, Universitätsverlag, 1990 (Konstanzer althistorische Vorträge und Forschungen 25). 
Alexandrescu, Petre et al., Histria VII. La Zone Sacrée d'époque grecque (fouilles 1915-1989), Bucarest/Paris, Editura Academiei Române/De Boccard, 2005.

Alexandrescu Vianu, Maria, Histria IX. Les statues et les reliefs en pierre, Bucarest/Paris, Editura Enciclopedică/De Boccard, 2000. —, "Théos Mégas», Dacia N. S., 43-45 (1999-2001), p. 73-78.

Avram, Alexandru, "Histria», in Ancient Greek Colonies in the Black Sea, ed. by Dimitrios V. Grammenos, Elias K. Petropoulos, Thessaloniki, Archaeological Institute of Northern Greece, 2003, vol. I, p. 279-340.

—, «Le corpus des inscriptions d'Istros revisité», Dacia N. S., 51 (2007), p. 79-132.

Avram, Alexandru, Bîrzescu, Iulian, Zimmermann, Konrad, «Die apollinische Trias von Histria", in Kult(ur)kontakte. Apollon in Milet-Didyma, Histria, Myus, Naukratis und aufZypern. Akten der Table Ronde in Mainz vom 11.-12. März 2004, hrsg. von Renate Bol, Ursula Höckmann, Patrick Schollmeyer, Rahden, Leidorf, 2008, p. 107-144 (Internationale Archäologie 11).

Avram, Alexandru, Zimmermann, Konrad, Mărgineanu Cârstoiv, Monica, Bîrzescu, Iulian, "Nouvelles données sur la Zone Sacrée d'Histria", in Une koinè pontique. Cités grecques, sociétés indigènes et empires mondiaux sur le littoral nord de la mer Noire (VII e s. a.C. $-I I I^{e}$ s. p.C.). Actes du colloque de Bordeaux, 14-16 novembre 2002, éd. par Alain Bresson, Askold Ivantchik, JeanLouis Ferrary, Bordeaux, Ausonius, 2007, p. 241-249 (Ausonius, Mémoires 18).

Bîrzescu, Iulian, "Histria. Graffiti din "Zona Sacră”. Dedicaţii către divinităţi», Studii ş̧ cercetări de istorie veche şi arheologie, 54-56 (2003-2005), p. 201-210.

—, "Some Ceramic Inscriptions from Istrian Sanctuaries: the Naukratis Approach", in Naukratis: Greek Diversity in Egypt. Studies on East Greek Pottery and Exchange in the Eastern Mediterranean, ed. by Udo Schlotzhauer, Alexandra Villing, London, British Museum Press, 2006, p. 169-174.

Bordenache, Gabriella, Pippidi, Dionisie Mihai, «Le temple de Orò $\varsigma$

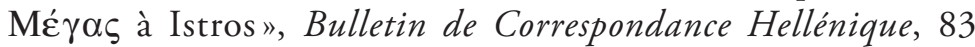
(1959), p. 455-465. 
Höckmann, Olaf, Peschel, Gerald J., Woenl, Anja, «Zur Lage des Hafens von Histria. Die Prospektionskampagne von 1996", Dacia N. S., 40-42 (1996-1998), p. 55-102.

Mărgineanu CÂrstoiu, Monica, "Plans de villes romaines en Mésie inférieure", in Bauplanung und Bautheorie der Antike, hrsg. von Wolfram Hoepfner, Berlin, Deutsches Archäologisches Institut, 1983, p. 297-315 (Diskussionen zur archäologischen Bauforschung 4).

—, "Der Theos-Megas-Tempel von Histria. Die Architektur", Dacia N. S., 33 (1989), p. 79-110.

—, "Bauelemente des Theos-Megas-Tempels von Histria", in Bautechnik der Antike, hrsg. von Adolf Hoffmann, Ernst-Ludwig Schwandner, Wolfram Hoepfner, Gunnar Brands, Mainz, Philipp von Zabern, 1991, p. 39-58 (Diskussionen zur archäologischen Bauforschung 5).

-, Histria XII. Architecture grecque et romaine. Membra disiecta. Géométrie et architecture, Bucureşti, Editura Academiei Române, 2006.

Rusjaeva, Anna Stanislavovna et al., Drevnejšij temenos Ol'vii Pontijskoj [Le plus ancien téménos d'Olbia du Pont], Simferopol', Centr arkheologičeskikh issledovanij BF «Demetra», 2006 (Materialy po arkheologii, istorii i ètnografii Tavrii, Supplementum 2).

Silant'eva, Pelageja Fjodorovna, Terrakoty Pantikapeja [Les terres cuites de Panticapée] = Terrakotovye statuètki III. Pantikapej [Statuettes en terre cuite III. Panticapée], Marija Mikhajlovna Kobylina (otv. red.), Moskva, Nauka, 1974, vol. III, p. 5-37 (Svod arkheologičeskikh istočnikov G 1-11, obščaja redakcija: B. A. Rybakov).

Vecchio, Luigi, "Medici e medicina ad Elea-Velia", in Elea-Velia. Le nuove ricerche. Atti del Convegno di Studi, Napoli, 14 dicembre 2001, a cura di Giovanna Greco, Pozzuoli, Naus, 2003, p. $237-$ 269 (Quaderni del Centro di Studi sulla Magna Grecia 1).

Vinogradov, Jurij G[ermanovič], "Heilkundige Eleaten in den Schwarzmeergründungen", in Bürgersinn und staatliche Macht in Antike und Gegenwart. Festschrift für Wolfgang Schuller zum 65. Geburtstag, hrsg. von Martin Dreher, Konstanz am Bodensee, Universitätsverlag, 2000, p. 133-149. 
Zimmermann, Konrad, "Ausgrabungen in der Tempelzone von Histria", Ethnographisch-archäologische Zeitschrift, 22 (1981), p. 453-467.

—, "Zu den Dachterrakotten griechischer Zeit aus Histria», in Histria. Eine Griechenstadt an der rumänischen Schwarzmeerküste, hrsg. von Petre Alexandrescu, Wolfgang Schuller, Konstanz am Bodensee, Universitätsverlag, 1990, p. 155-177 (Konstanzer althistorische Vorträge und Forschungen 25).

—, "Archaische Dachterrakotten aus Histria", Hesperia, 59 (1990), p. 223-233.

—, "Griechische Altäre in der Tempelzone von Histria», in L'espace sacrificiel dans les civilisations méditerranéennes de l'Antiquité. Actes du Colloque tenu à la Maison de l'Orient, Lyon, 4-7 juin 1988, éd. par Roland Etienne, Marie-Thérèse Le Dinahet, Paris, De Boccard, 1991, p. 147-154 (Publications de la Bibliothèque Salomon-Reinach 5).

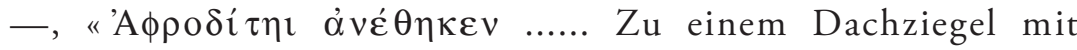
Votivinschrift", in Civilisation grecque et cultures antiques périphériques. Hommage à Petre Alexandrescu à son $70^{e}$ anniversaire, éd. par Alexandru Avram, Mircea Babeş, Bucureşti, Editura enciclopedică, 2000, p. 239-251.

\section{Crédits iconographiques}

Fig. 1:

Architecte Anişoara Sion.

Fig. 2:

D’après Alexandrescu, Petre et al., Histria VII. La Zone Sacrée d'époque grecque (fouilles 1915-1989), Bucarest/Paris, Editura Academiei Române/De Boccard, 2005.

Fig. 3, 4, 7-8 et pl. XXXV-XXXVI, XXXVIII-XL:

Photos Iulian Bîrzescu.

Fig. 5:

Iulian Bîrzescu, architecte Monica Mărgineanu Cârstoiu. 
Fig. 6, 9 et pl. XXXIX.1 :

Dessins Argeş Epure.

Pl. XXXVII :

Dessin Florina Panait-Bîrzescu. 